\title{
ARTIFICIAL INTELLIGENCE, AUTOMATION, AND INTEGRATED CONSTRUCTION
}

by

\author{
Photios G. Ioannou and Robert I. Carr \\ University of Michigan \\ Ann Arbor, Michigan 48109-2125, USA
}

\section{Introduction}

The United States spends $\$ 300$ billion a year, about $8 \%$ of the gross national product on new construction. A comparable amount is spent on interest for construction loans, and even more is spent for operation expenses. Given the magnitude of these expenditures, there is a pressing need for better efficiency, lower costs, and improved quality in the constructed product.

The fragmentation of the industry and the conservatism and slow adaptation of technology, as well as trends in the international arena coupled with technological advancements in the information and automation fields, point toward a framework for vertical and horizontal integration and construction automation as probably the most promising solutions to this problem.

Integrated construction is envisioned as a cooperative environment for facility planning, design, construction, operation, and use, in which each participating decision maker contributes and receives information to and from other participants so that each entity's decisions at every project level are cognizant of the needs of others and are always based on the most recent, complete and reliable information available to the group. This paper describes the roles of artificial intelligence and design and construction automation in achieving integrated construction. 


\section{Background - Problem Definition}

The delivery process for a facility involves a unique, ad hoc grouping of entities that plan, design, construct, operate, and use the facility, typically over a planned 50 year time span. For most parties, the facility is one of an intermittent stream of concurrent facilities which they plan, design, construct, operate, or use. Each party has its own organizational and information infrastructure which gives it continuity from one facility and time frame to another. The parties are bound together and work together using information links, through which they communicate and share information.

Construction is an information intensive industry, whose project information costs typically equal the on-site labor costs, a fact that often goes unnoticed by the casual observer. Every stage in the life cycle of a facility requires decision making that depends on the quality and amount of information currently available. Every decision creates information (data, conditions and constraints) that is part of the input to another decision in the same or other discipline, that may or may not necessarily follow the first in chronological order. Construction decisions are highly interdependent throughout the project delivery process, both from a time and organizational point of view. As such, they are very difficult to streamline and optimize in a non-iterative framework.

To illustrate, let us consider the flow of information related to structural steel for a building. The architectural layout of a building dictates the amount of open space and the location of partitions and hence constrains the placement and size of the vertical and horizontal support members. The relationship also holds in reverse. The amount of space and its function also determine the amount of duct work for the HVAC system which must be carried within the available concealed ceiling area without obstructing or being obstructed by structural members. The structural steel member sizes depend on the choice for vertical and horizontal member type, connection types, geometry, and load conditions based on dead and live load conditions. The configuration of the superstructure and the vertical and horizontal loads in the columns influence the choice of the foundation type and its design. Once these design decisions are made, the information concerning the preferred layout must be documented using drawings and 
schedules and submitted as part of the drawings to the contractor who must then estimate the cost of steel, determine the method and sequence of erection, and convey all relevant information to the steel fabricator. The fabricator must in turn prepare the shop drawings for the individual members and their connections; submit them for approval to the contractor and the designer; cut, shape, drill, bolt, and weld all shop connections; and deliver the partly assembled parts to the contractor for field erection using the design specifications and the contractor's instructions. The contractor must plan the time-phased delivery of steel members to the site, their temporary storage and handling, and integrate their erection by allocating the necessary resources (labor, equipment, funds and time) within the overall construction process schedule.

Some typical problems with the current design-construction process are:

- There is no complete recognition of interrelationships among decisions across disciplines and time frames.

- Parties operate independently and do not share all information crucial to decision making.

- Misinterpretations of design intent are possible.

- The most current drawings and specifications and other data are not always available, or used.

- Changes in plans and specifications can be initiated without informing others who may be currently using older versions.

- Design decisions are not always based on the best cost (materials, methods, \$/unit) information available.

- Objectives such as constructibility, maintainability, operability, and livability are not directly considered. 
In summary, the fragmentation of the industry, the lack of responsive information links, and the lack of effective cooperation among the project participants are the major problems that need to be addressed within the framework of integrated construction.

\section{Integrated Construction: A framework for cooperative decision optimization.}

Each civil engineering construction project is undertaken by a unique group of participant entities representing nodes in a network which communicate through information links. The structure of this network is very similar to a telecommunications network in which information flows within or across organizations and time frames.

At the highest conceptual level the basic objective of an integrated system is to allow all parties to have immediate access to the best information available within the network in order to cooperatively make optimal decisions, given the existing knowledge and expertise in the industry and the prevailing physical, legal, and regulatory constraints which influence the group. The interrelationships of decisions during design and construction is illustrated in Figure 1.

Consequently, integrated construction requires an industry-wide information architecture that facilitates the support of each project by a compatible linkage of randomly selected, interchangeable, independent nodes, each selected to play a specific role and to cooperate with others toward the realization, operation, and use of the facility. Each project will be the product of a group which uses an integrated but distributed set of decision support systems that are based on a life-cycle project database. This is the information realization of the facility, in the same way that the completed facility is its physical realization.

Essential to the full realization of integrated construction is an on-line, direct access logical information system among facility participants. In principle, this requires that:

1. The information required for the parties' decision and operation systems are automated so they can be responsive in real time, and 


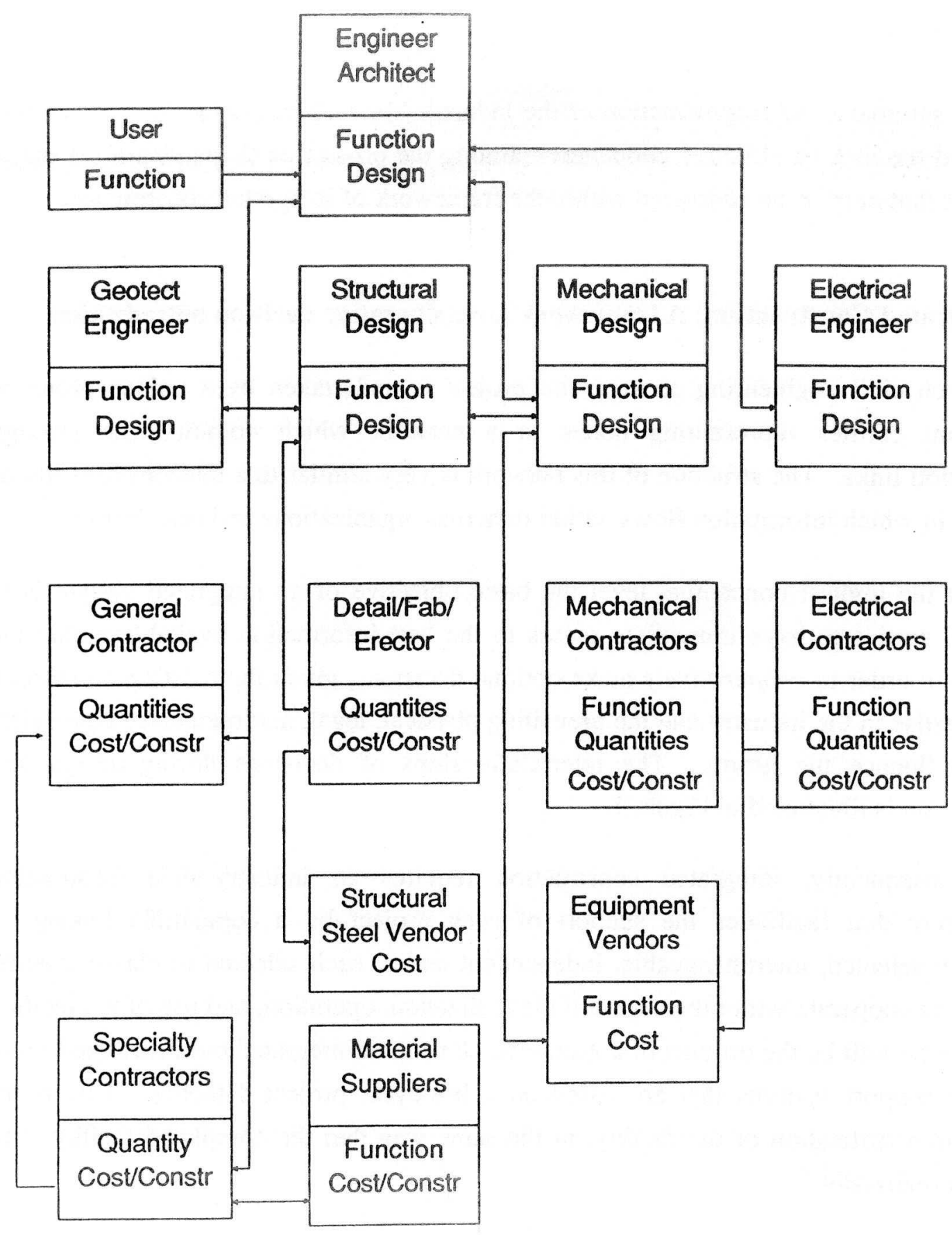

Figure 1: Information Flow for Integrated Project 
2. The facility and industry information infrastructure provide real-time, interactive demand access among randomly selected, independent parties to provide a universal information storage and retrieval architecture.

\section{Integrated Construction - System Architecture}

The realization of an integrated construction environment, as described above, requires an enormous amount of research and development at both the conceptual and implementation levels. The number of topics that need to be examined is so large that it is impossible for this paper to present an exhaustive list.

The relationships among project participants and some of the major research needs involving the communication and cooperation of multiple entities are shown in Figure 2. These are some of the issues that must be resolved within the communications and information exchange framework of construction integration.

At a more basic level, integration requires automated decision support systems for each of the node entities within the project group. Figure 3 shows the fundamental elements of a generalized decision support framework that may be used by a project participant to tie into the project information network. The central concept illustrated by this model is that each decision making entity both consumes and produces information. An example application of this model to a decision support system for structural design is shown in Figure 4.

The central repository of project information is an integrated project database (IPDB) that represents the dynamic characterization of a facility during the project delivery process. The primary function of this database is to either hold or provide links to all the graphical and alphanumeric information required for the complete description of the facility at any point in time. The database should be coupled with a high-level shell for database maintenance and should provide data access to the distributed node application programs based on their subschemas. 


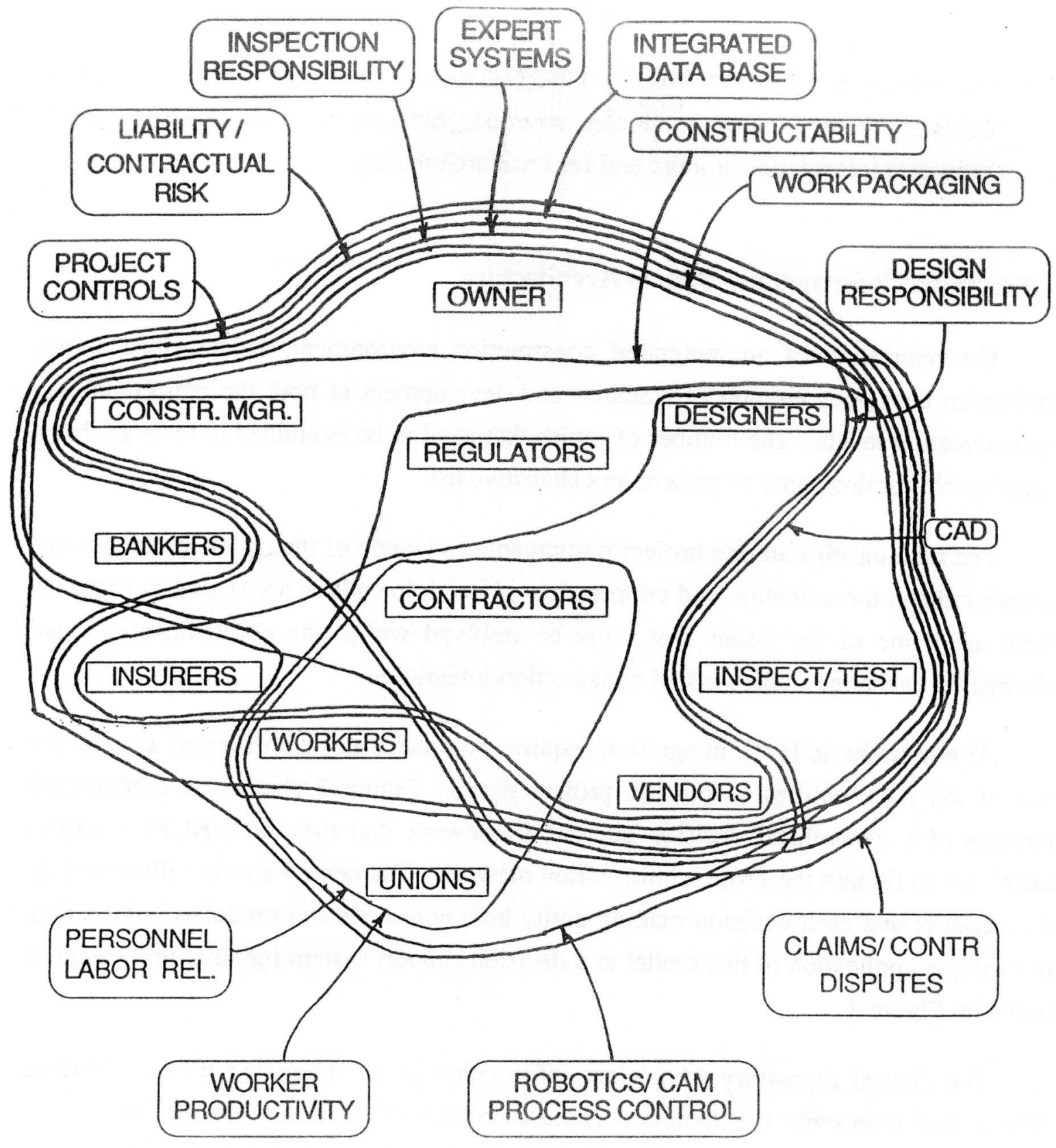

Figure 2: Relationships Among Project Participants and Some Major Research Needs 


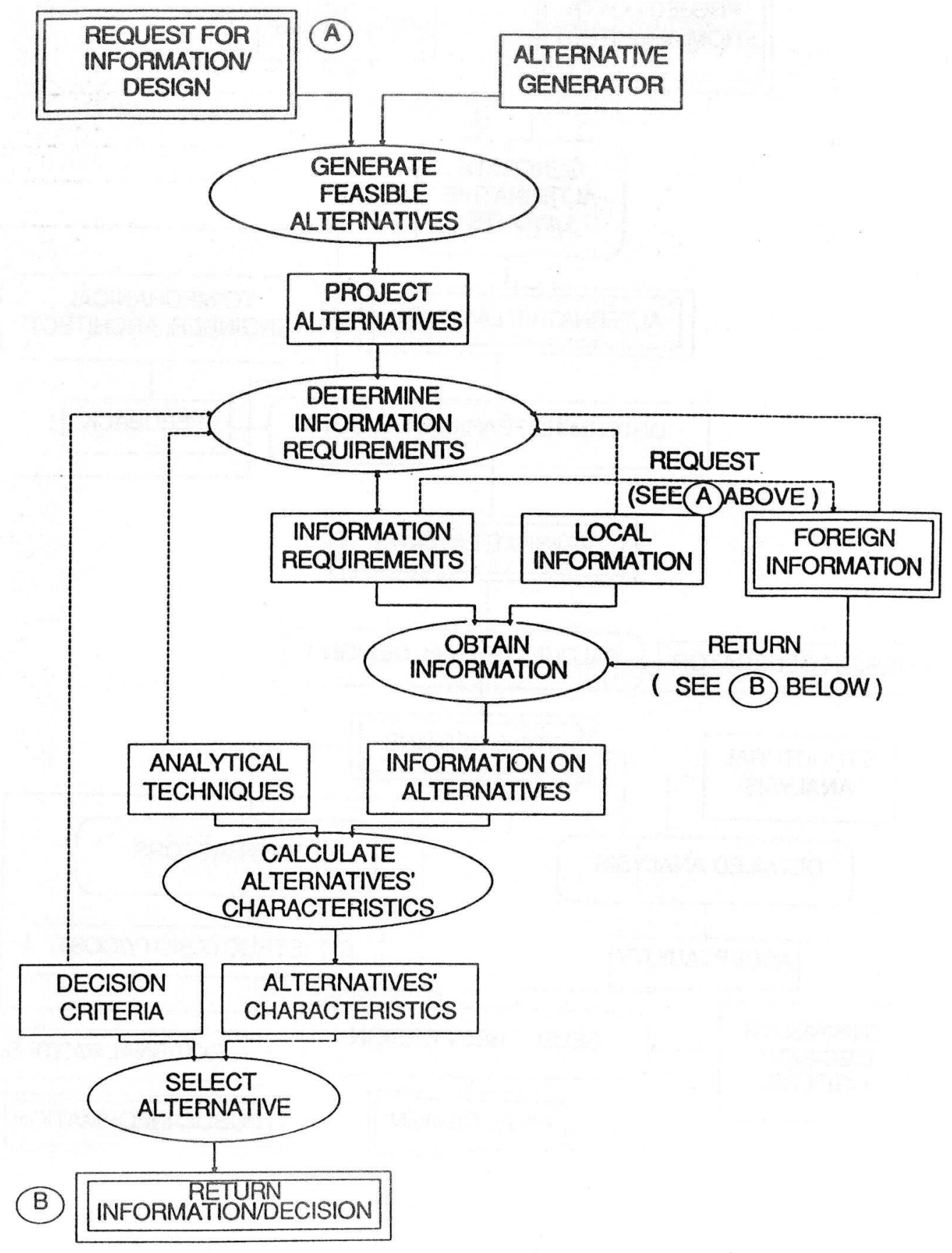

Figure 3: Model of Integrated Information/Decision Process 


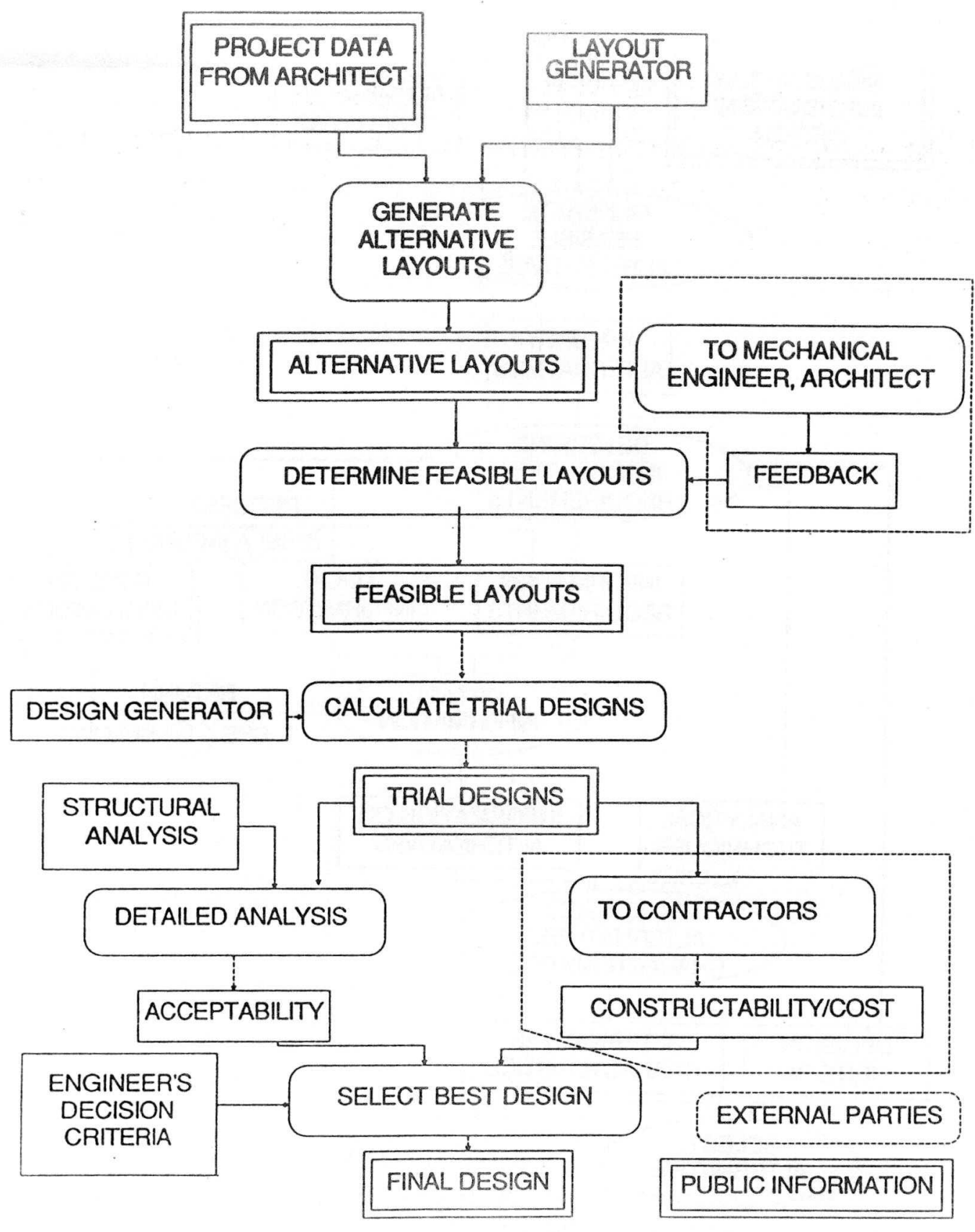

Figure 4: An Integrated Information/Decision Framework for Structural Design 
This is accomplished by first developing similar distributed node databases (DNDB) and communication-translation software for each of the project participants. The DNDBs are responsible for providing the input to applications software either through calls to the IPDB or through links to a participant's own central database (DCDB). In addition, they hold the data output of application programs and decision support systems used on a particular project and provide access to remote requests from the IPDB. The communication-translation software function as gateways (or active links) between the IPDB and the DNDBs and application programs. A schematic for a distributed node decision support system and its connections to DNDB and DCDB is shown in Figure 5. The general, many-to-many connectivity among participants and projects is illustrated in Figure 6.

The fundamental architecture of this model can be described as follows:

Each organizational entity participating in a project has its own central database (DCDB) which serves as the foundation of the in-house decision support systems which are built around it. These decision support systems derive their general knowledge base from the $\mathrm{DCDB}$, and they request project specific information from other project participants and outside agencies. This information is provided through the IPDB, either by active hooks (pointers and flags) or by argument-passing (values and flags) from the DCDB's of other organizations. Thus each distributed node has its own project database which contains the most up-to-date information that it has acquired externally or generated internally.

The integrated project database and its data manager shell serve as a repository and as a clearing house of information among the distributed nodes. In other words, the IPDB either holds the information needed by a participant or it has pointers to another participant's DNDB where the information can be found or generated. The degree to which the IPDB must be centralized as opposed to being distributed among the participants' DNDBs is one of the fundamental questions that must be examined. 


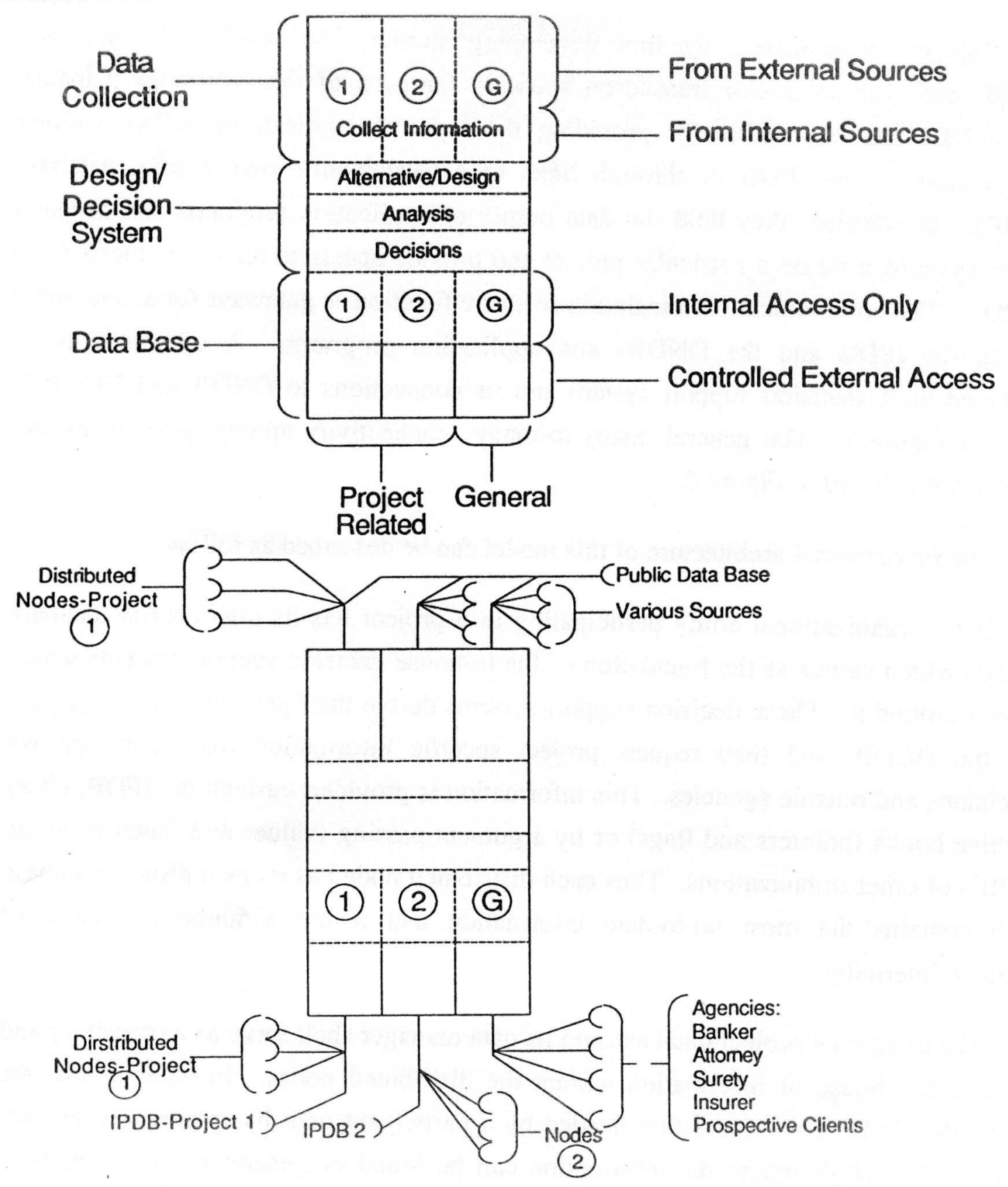

Figure 5: Model of Distributed Node Representative of a Project Participant 


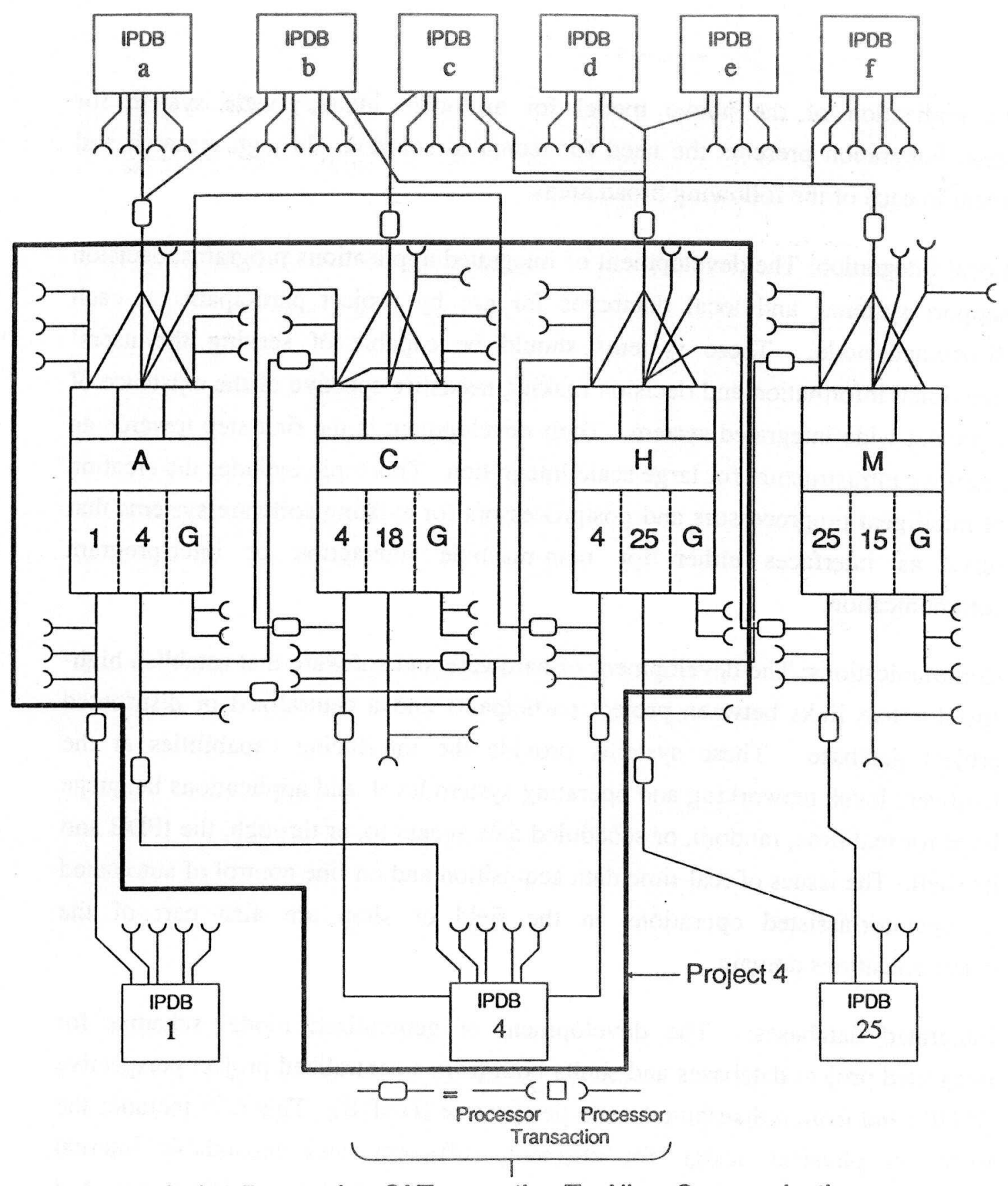

Active Processing Of Transaction To Allow Communication

Figure 6: Integrated Construction Information Architecture 
The realization of the above model for an open, industry-wide system for construction integration presents the need for technical solutions through research and development in each of the following broad areas:

1. Local integration: The development of integrated applications programs, decision support systems, and local databases for use by project participants at each distributed node. These systems should be capable of serving the users' functional information and decision making needs irrespective of the existence of a project-wide integrated system. Their development is the first step towards an interface infrastructure for large scale integration. This topic includes the creation of intelligent preprocessors and postprocessors for existing software systems that serve as interfaces either for man-machine interaction or inter-program communication.

2. Communications: The development of hardware and software that establish highspeed active links between project participants and a centralized or distributed project database. These systems provide the interfacing capabilities at the hardware level, networking and operating system level, and applications language level for real time, random, or scheduled data access to, or through, the IPDB and its shell. The issues of real-time data acquisition and on-line control of automated or operator-assisted operations in the field or shop are also part of the communications domain.

3. Integrated databases: The development of generalized model schemas for integrated project databases and shells both from a centralized project perspective (IPDB) and from a distributed node perspective (DNDB). This area includes the issues of physical design for efficient, sufficient, and expandable internal representation of graphical and alphanumeric data; logical design of schemas and subschemas; database command and definition languages; application and communication interfaces to IPDBs, DNDBs and DCDBs; data security; data integrity; etc. 


\section{Construction Integration, Computer Automation and Artificial Intelligence}

The field of artificial intelligence, and computer automation in general, plays a fundamental role in every aspect of the development of a general framework for integrated construction. The current state of the industry suffers from vertical and horizontal fragmentation and shows incompatibilities at all levels: from the computing hardware used by engineering and construction firms to the data structures and linguistic and graphical symbolism used by each discipline for input, output, or communications. These incompatibilities point to an immediate need for artificial intelligence applications in establishing a cooperative decision making environment.

Architectural and engineering design is a decision synthesis process that strives to achieve several objectives simultaneously (some of which are in direct conflict with each other) while satisfying external and internal constraints. Objectives such as aesthetics, function, cost, constructibility, safety, operability, and maintainability must be reasoned out and balanced. The difficulty in the joint consideration of these objectives often leads to an objective-to-constraint transformation in which the number of objectives is reduced by introducing acceptable levels for the objectives of secondary importance. This transforms the secondary objectives into constraints that must be satisfied. However, the problem of quantification of either the objectives or the constraints still remains and represents one of the most challenging areas of decision support research.

Some of the primary applications of artificial intelligence include:

1. Communications

- Intelligent interfaces and translators for hardware and operating and network systems.

- Knowledge based systems for network and systems interface configuration, monitoring, evaluation, and maintenance.

2. Local Integration

- Pre-processors and post-processors for existing and future applications software. 
- Intelligent assistants for man-machine interaction.

- Knowledge based modules within algorithmic design/construction decision support systems.

- Intelligent conversion of existing 2-D paper-based drawings to electronic 2-D or 3-D format.

- Intelligent conversion of electronic 2-D drawings to 3-D.

- Interpretation of symbolism in 3-D and the association of graphical objects with textual descriptions and attributes.

- Knowledge-based assistance for constructibility review during the design process. Rapid identification of potential problems and remedial action.

- Knowledge-based guidelines to promote and facilitate design for automated construction. Identification of automated construction requirements during design to set appropriate constraints and objectives.

- Dynamic evaluation of design performance as it is being developed at predefined or ad hoc intervals.

3. Global Integration

- Knowledge based systems for interfacing applications to databases within and across systems and disciplines.

- Intelligent search, retrieval, conversion, and compound information generation based on the specific data requirements for decision making of each project participant.

- Knowledge based systems for identifying cause and effect relationships among design decisions across disciplines.

- Knowledge based systems for identifying cause and effect relationships among design and construction decisions and among design/construction and maintenance/operations.

- Knowledge based assistance for the evaluation of the integrated designconstruction system at the completion of a project. 


\section{Construction Integration and Construction Automation}

The benefits of construction integration stem primarily from the value of the information and knowledge that is made directly available to the participating decision makers. Thus, integration has value because it will lead to better planning, design, and construction decisions.

Construction integration, however, has additional benefits that are not immediately apparent. It allows the direct consideration of decision cross-impacts within the project organizational structure. For example, it identifies conflicts and interferences among the several disciplines involved in the contractual hierarchy for the design, and/or the interaction among construction management, the general contractor, the various subcontractors, fabricators, materials suppliers, and regulatory agencies.

In this respect, construction integration provides a fertile infrastructure for the development and wide spread adoption of construction automation. Construction automation requires an electronic description of the project elements, their spatial and connectivity relationships, their material and surface properties, and the field operating conditions. Furthermore, automation requires the development of and input to support systems such as expert systems, simulation, optimization, and construction CADD/CAM systems for the automated generation of robot operation and control programs and instructions for human action. The development of these systems is also required for construction integration. Integration and automation also require the development of methods for the acquisition of as-built information during the construction phase, for design evaluation, input to facility evaluation, operation, maintenance, and construction planning and control.

Expert systems allow the automatic generation of control programs (or manual task designs) for specific project operations. Simulation provides an automated test-bed for analyzing and costing construction operations described in control programs, to provide design and construction planning feedback. Optimization techniques select the best of alternative, feasible control programs. CADD/CAM provides the automated, interactive 
graphical and object oriented data base representation required in a construction planning environment.

Essential to a contractor's successful automation of construction operations is the integration of construction automation into the contractor's information organization. Automated development of control programs, coupled with simulation, allows accurate estimation of operation costs and optimization of construction operations. Automated real time data acquisition and analysis during operations provides necessary real time control of automated operations, and it also provides real time cost control to the contractor's management information system. Going one step further, the data can also provide the base for dynamic programming of microprocessor controlled servo mechanisms for automated equipment operation. That is, the control program modifies itself in real time to fit the operating conditions actually encountered in the field.

\section{Emerging Technologies for Integrated Construction}

It is evident that even though the current state of the art in electronic data information systems is in the midst of an unprecedented revolution, the goal of a totally integrated construction industry must follow a slower route while the required information infrastructure for design and construction is developed, tested, improved, and accepted by the industry.

Described below are some of the emerging technologies that, at least in the short run, appear promising in providing an improved level of integration in the industry.

\section{Optical Fiber Networks - Hardware Integration}

CADD/CAM files require a digital communication format and high speed transmission rates of $100 \mathrm{Mbits} / \mathrm{sec}$ (Mbps). Fiber technology is used for high-speed token-ring networks under the Fiber Distributed Data Interface (FDDI), a new IEEE standard for 100 Mbps token-ring LANs. Such networks can function as the backbone for connecting smaller twisted pair or coaxial cable ring networks or minicomputers and mainframes. FDDI is expected to support voice, data, and video to provide enough 
bandwidth for graphics workstations and access to large-capacity, mainframe-based databases. A fiber optic version of the Manufacturing Automation Protocol (MAP) is currently being developed by a IEEE task force. A MAP network might use a starshaped system with 32 ports and a maximum distance between nodes of $1 \mathrm{Km}$.

OSI (Open System Interconnection) is a similar network standardization that is advocated on an international scale by ISO (the International Organization for Standardization which is primarily concerned with general standardization, documentation, and design standards) and IEC (the International Electrotechnical Council which is concerned with electrical, electronic, and related product safety). The proposed OSI promotes standardization along seven hierarchical layers of communications ranging form the physical networking hardware at the nodes and links to the application level, which includes graphics, CADD/CAM/CAE, and robotics.

\section{Expert Systems for Network Management/Configuration}

Current estimates indicate that shortage of skilled personnel leaves $30 \%$ of network management positions unfilled. This figure may reach $50 \%$ by 1990 . Companies struggling to cope with a growing shortage of skilled network managers and support personnel may find relief in a new breed of expert systems software for network management which is shortly expected to enter the commercial market. Though currently available network-monitoring systems merely collect symptoms and issue alerts, the announced expert systems can store and use information about the network topology, database designs, and application programs. Managers can monitor network performance and simulate changes in the system configuration to predict their impact. More sophisticated products now being developed will allow the overseeing and control of network equipment from multiple manufacturers. A dedicated LISP machine-based expert system interprets events reported by the monitor and recommends a course of action to the network operator. The proliferation of hardware and networking systems and the shortage of qualified personnel makes network configuration and management one of the optimal applications of artificial intelligence. 


\section{Promising Alternatives to Paper-based Plans and Specs}

Civil engineering design and construction are information intensive disciplines that require the means to store and access massive amounts of graphical and alphanumeric information. Today the means are typically paper, film, and electronic media that include not only the classic computerized database processing systems, but also micrographics.

Micrographics dramatically reduces the size of documents and drawings and transforms them into easily stored images. It has already been successfully employed for many years and will continue to evolve and serve a useful purpose in information management. Its new uses involve the direct connection of automated micrographics storage and retrieval (SAR) devices within a computing environment. The principal technology uses microfilm strips mounted on apertures within paper cards that contain record information and indexing. Aperture cards, which resemble the punched cards of the sixties, can be automatically retrieved under computer control, copied, reproduced on paper and film, and digitized into raster images for on-line editing and merging into CADD.

Another important technology is the laser optical disk (LOD). There are three fundamental types: the compact disk read-only-memory (CD-ROM); the write-once, read-mostly (WORM); and the erasable optical disk (EOD).

CD-ROM's have information transcribed on the medium through a mastering process. Typically, an organization produces a master disk or tape which is then sent to a third company where multiple identical CD's are pressed in a process analogous to the phonograph industry. As a result, CD's are most appropriate for storing information that is not dynamic and for which a large audience exists. Codes would be a good example. Typical CD's are single sided, have a diameter of 4.72 inches $(12 \mathrm{~cm})$, and hold about 500 Mbytes of data.

WORM technology allows writing information on an optical disk in a similar way as magnetic media. Once the information is written on a WORM disk it can be read repeatedly, but it cannot be erased or changed. EOD disks can be written, erased, and rewritten exactly like magnetic disks. Reading and writing transfer speeds are in the order 
of 200-500 Kbps. Current best estimates for media life is $15-20$ years. WORM and EOD disks have a different physical format from CD-ROM disks with standard diameter sizes of $3.5,5.25,8,12$ and 14 inches. The larger sizes, especially $12 \mathrm{inch}$, have significant cost advantages over the smaller ones because they are double sided, hold more data, and can be readily used with automatic disc-handling devices. These devices resemble phonograph juke-boxes that can retrieve information from any of hundreds of 12 inch disks without human intervention. This expands the on-line storage capability of the device to hundreds of Gbytes. Similar juke-boxes are being developed for 5.25 inch disks.

Micrographics and optical disks represent a less dynamic but less expensive and more stable information medium for graphical information storage, retrieval and transfer. Microfilm is currently the cheapest option for archival storage and reproduction. Even though it has much slower retrieval speeds when compared to other electronic media it has a much longer life. However, it is unsuitable for use during design, because extractions, modifications and development of new designs still involves a significant amount of manual effort. Microfilm mounted on aperture cards is an excellent solution for long term storage, and, given its effective resolution of well over a $1000 \mathrm{dpi}$, it can be easily converted through scanning to digital form at 200-400 dpi for editing and storage on optical disks.

Optical disks can store data, graphics, voice and video information in a standard file protocol, and have unique advantages that promise to make the technology an instant success (the demand for the recently announced WORM drives by IBM is four times larger than originally estimated). Three standards in this area have already been developed on an international scale and an optimal configuration is very likely to prevail before the technology becomes widely available. These standards are: CD supported by Sony, Philips, Hitachi, MicroSoft and other software companies; CD-I supported by Sony and Philips; and DVI recently announced by GE-RCA.

The WORM technology is particularly attractive because the data cannot be altered once it is written. As a result, WORM disks are the closest electronic alternative to paper, given the current state of the industry from a contractual and legal liability 
perspective. Their cost and reproduction time are minimal, and their capacity is phenomenal. The paperless construction communications of the near future could be achieved either physically (e.g. here are the plans and specs on a $500 \mathrm{MB}$ disk) or electronically (e.g. by downloading one WORM disk on another over a network).

\section{An Example of Text and Graphics Integration in Industry}

The potential for text and graphics integration is best illustrated by recent examples in the newspaper industry. Because of the scarcity of qualified graphics artists, a newspaper chain (Knight-Ridder) has set up a network for sharing graphical information among all its newspapers. In the process, it has also developed a new business venture for selling its graphics outside the chain. To accomplish this goal, they made use of BusinessTalk a new service from General Electric Information Service Co. (GEISCO) for companies that require wide-area internal communications, communications with dealer networks, or a platform for a new service enterprise (like the Night-Ridder Graphics Network). BusinessTalk differs from similar services offered by a number of information and videotex companies in the degree of integration between the service and the users' front-end hardware. Users must be equipped with Macintosh or IBM PC's and with software provided by GEISCO to communicate with mainframe-based system services using + proprietary communications protocols. The result is what appears as a seamless system for e-mail, file transfers, bulletin boards and text database management. Capitalizing on the worldwide communications capability of the system, the NightRidder Graphics Network allows the subscribing newspapers to retrieve McDraw graphics from a daily listing of chronological or geographical area libraries on GEISCO's mainframes. Users can manipulate these graphics with McDraw on the Macintosh before sending them to a PostScript output device for publication. In terms of pricing and payments the service mimics the text-based wire services already familiar to the users, and it has proven to be successful with both large and small newspapers. Even though they receive no fee, artists from the client companies also make contributions to the libraries. These are acknowledged if used in other publications making the system a twoway cooperation process. Associated Press is also developing a similar system. 


\section{Conclusion}

The integration of information within each of the phases in the project delivery process - planning, design, construction, operation and maintenance - has already begun. The migration from manual processes to computer-based automated and integrated systems within owner, designer, and contractor organizations is also well under way. These are the first steps toward the partial and later the complete integration of the construction industry at the project level.

Obviously, the development path toward an environment for integrated construction, as well as its final form and capabilities, will be directly influenced by organizational, legal, contractual, motivational, technical and market variables. The impact of these variables cannot be preassessed. We can only predict that the process will probably be evolutionary rather than revolutionary.

At this stage, the single most important objective in integrated construction research is the development of a fundamental system design for information interchange that is sufficiently modular to easily incorporate new input, output, storage and communications hardware and applications software. Irrespective of the rapid pace of technological change, the fact remains that modern engineering and construction companies have and will make considerable investments in manual and computer systems. Tying them together in a smooth upgrade plan will always be an ongoing challenge. The electronic age has already begun, and the advent of integration is inevitable. 